\title{
On the influence of inter-layer time and energy density on selected critical-to- quality properties of PA12 parts produced via Laser Sintering
}

\author{
M. Pavan*1,2, M. Faes*,1, D. Strobbe ${ }^{1}$, B. Van Hooreweder ${ }^{1}$, T. Craeghs ${ }^{2}$, D. Moens ${ }^{1}$, W. \\ Dewulf $^{1}$ \\ ${ }^{1}$ KU Leuven, Department of Mechanical Engineering \\ ${ }^{2}$ Materialise NV, corporate R\&D, HQ Leuven Belgium \\ * both authors contributed equally to the work
}

\begin{abstract}
Laser Sintering (LS) of polymers is an Additive Manufacturing technique progressively used to produce functional parts. However, LS parts still present a certain quality variability. Efficient consolidation of the polymeric powder, necessary to obtain a dense part, mainly depends on the viscosity of the molten polymer and on the time given for the polymer to coalesce. The polymer viscosity is a function of the temperature and, consequently, of the energy density (ED) input by the laser. The sintering time strongly depends on the surface area to be laser-scanned in each layer, which in turn is strongly related to the product complexity and the number of parts within one build. The aim of this work is to investigate how this thermo-temporal effect, influenced by the ED and the inter-layer time, influences the resulting LS PA12 part quality at both micro-level (e.g., porosity, crystallinity) and macro-level (e.g., dimensional accuracy, mechanical performance).
\end{abstract}

Keywords: Laser Sintering; mechanical performance; inter-layer time; energy density; dimensional accuracy; porosity measurement.

\section{Introduction}

Due to the low time-to-market, large design freedom and efficient use of production resources, Additive Manufacturing (AM) receives an increasing industrial interest, especially in sectors which require high quality standards, such as e.g. the medical and automotive sectors. Offering a good compromise between dimensional accuracy and mechanical performance, Laser Sintering (LS) is one of the most promising AM processes for polymers [1]. Due to the layer-by-layer nature 
of this process, complexity can be added to the design without increasing the cost of the production. Figure 1 shows a typical layout of a Laser Sintering machine. To date, different commercial machines exist, which differ in e.g. laser optics, powder feeding and deposition mechanisms, build volume or means of preheating [2-5].

\section{Figure 1}

Due to the current trend of increasing use of LS parts in functional applications, critical-to-quality (CTQ) parameters of the produced parts such as the porosity, crystallinity, dimensional accuracy, and mechanical performance need to be assessed critically. A commonly used factor influencing these parameters is the laser energy density (ED) used to produce the part, which is formally defined as [3]:

$$
E D=\frac{P}{H D \times S S}
$$

with $P$ the power of the laser beam, $H D$ the hatch distance, which is the distance between two adjacent parallel scan vectors, and $S S$ the scan speed of the laser. A commonly known disadvantage of PA12 components produced using LS is the presence of residual porosity, which measures typically around 3-5\% [6-7]. These pores are mainly the result of insufficient consolidation, and are formed as a result of the fact that the time to achieve full coalescence and consolidation of the molten particles is much longer than the inter-layer time (i.e. the time between the scanning of a certain point of the layer and the recoating operation). The ideal coalescence between two spherical particles is described by the Frenkel's model [8], which relates the coalescence dynamic to the surface tension $(\gamma)$ and the zero-shear viscosity $\left(\eta_{0}\right)$ of the molten polymer at a given temperature:

$$
\frac{x}{r}=\left(\frac{3 \gamma}{2 \eta_{0} r}\right)^{\frac{1}{2}} t^{\frac{1}{2}}
$$

where with $x$ is the formed neck radius between the particles, $r$ isthe original radius of the particles and $t$ isthe sintering time. The zero-shear viscosity of the particles in molten state increases when the temperature decreases which, according to (2), requires a longer sintering time for the same level of particle-coalescence. Basically, the degree of consolidation achieved for a certain 
polymeric material depends on the polymer thermal history in the molten state, which depends on the scanning strategy (i.e. scanning pattern and process parameters) used during the process [6, 9]. These residual pores act as local stress concentrators in the LS component and, therefore, have a detrimental effect on the elastestatic performance ultimate tensile stress and strain at failure, especially when specimens are loaded along the printing direction [10]. Van Hooreweder et al. [11-13] showed in this context how under certain cyclic mechanical loading conditions, failure is caused by both mechanical and thermal loading. The same research indicates that the nucleation of the cracks often occurs due to unmolten particles located in the middle of the specimen. Although Van Hooreweder [13] indicates that the material density is a crucial factor influencing the fatigue life of LS PA12 components, this influence was attributed to a higher number of unmolten powder particles and consequently a higher chance of crack initiation. However, the The presence of the pores was reported to not significatively-influence the fatigue performance significantly [10-12]. This could be due to the difficulties to separate the contributions to the fatigue failure of the thermal and mechanical effects, with the latter intuitively linked to the porosity content, as the stress concentration around pores could act as crack initiators. The influence of the ED on the mechanical properties has widely been reported in literature [4-5, 10, 13-15 14-16]. It is generally accepted that a higher ED leads to higher elastostatic mechanical elastic properties such as Young's modulus and tensile strength. This is mainly attributed to the improved coalescence resulting from the higher temperatures occurring in polymer melt when higher EDs are used. However, the positive correlation is only valid as long as no thermal degradation occurs. Recently Faes et al. [16-17 17-18] studied heterogeneity in the mechanical properties of LS-PA12 parts by using Digital Image Correlation (DIC) to measure the entire strain field during a tensile test. They found local higher strains at those locations where other parts were built simultaneously with the part that was being tested, assuming that this is due to an increase in inter-layer time, but no quantitative results were presented to confirm this idea.

The mechanical properties of LS-PA12 parts are also influenced by the amount of crystalline phase in the microstructure. Higher crystallinity leads to an increase in Young's modulus and tensile strength, while the elongation at break tends to decrease. The amount of crystalline phase depends on the thermal history of the material, which is mainly determined by the applied process parameters. Zarringhalam et al. [198] showed how the crystallinity present in LS-PA12 parts increases when the ED delivered is below a certain value, due to the residual unmolten material in 
the core of the particles. Gogolewski et al. [19 20] showed how prolonged annealing at a higher temperature increases the crystallinity of PA12. The annealing temperature and time investigated in their study are comparable to those of the LS process, suggesting that the permanence of the parts in the building envelope determines a progressive increase of their crystallinity, depending on their position within the build. Verbelen et al. [210] reported dilatometry measurements on different PA12 powders showing reduction in the specific volume during the crystallization phase ranging from $3.9 \%$ until $4.7 \%$. Different crystallinity translates in a different material shrinkage, which might lead to different final part dimensions.

The dimensional accuracy of LS-PA12 parts is influenced by numerous process parameters [ $Z 1$ 23-22-24]. In this context, Strano et al. [23z] showed that the first layer is thicker than subsequent layers due to different flowing conditions on loose powder as compared to on an already-sintered material. Seepersad et al. [234] investigated how the wall thickness influences the tolerances of moving parts. Local dimensional deviations suggest the presence of a local rise in temperature, which leads to an extra sintered region. Different local heat flow conditions linked to the part geometry contribute in determining the magnitude of the effect. Other parameters influencing the local temperature are the inter-layer time and the laser ED input to print the part, which are in an industrial context tuned to achieve better part quality and/or decrease the printing time. Since longer printing times lead to higher production costs, nesting algorithms for optimally placing different parts in a single build volume are commonly used to optimize the machine usage [254]. The final placement of the parts in the build defines the amount of surface to be laser-scanned for each layer, which directly affects the inter-layer time. Another parameter affecting both inter-layer time and the ED is the scanning strategy, which defines laser-paths, the laser scanning speed and the ED locally delivered to the platform.

To the knowledge of the authors, the combined effect of the scanning area (and consequently interlayer time) with different ED input has not been studied yet in literature. The goal of this research paper is, therefore, to assess this combined effect on critical-to-quality parameters such as dimensional accuracy and mechanical strength, supported by a more fundamental study on the local degree of porosity and degree of crystallinity.

\section{Methods}




\subsection{Sample production, machine, build envelope}

The test samples were produced using a P396 machine from EOS GmbH using a PA2200 PA12 powder with a mixing ratio 50/50 between virgin and recycled powder and an alternate $\mathrm{x}-\mathrm{y}$ scanning pattern. Figure $2 \mathrm{~b}$ shows the configuration of the build, where 25 tensile bars (ASTM D638 type III) have been printed upright using 5 different ED levels (5 specimens per level), corresponding to the process parameters reported in Table 1. All specimens were produced with the same slicing offset and rescaling parameters. To study the influence of the inter-layer time, the gauge length $(50 \mathrm{~mm})$ of the tensile bars was divided in 5 different regions and the relative area to be laser-scanned was changed by adding sacrificial parts, as also shown in Figure $2 b$. The sacrificial parts were placed close to the corners of the platform to eliminate local thermal influence on the tensile bars. The amount of extra scanning area introduced by the sacrificial parts ranged between 0 and $200 \mathrm{~cm}^{2}$, with discrete variations between different regions being a multiple of 50 $\mathrm{cm}^{2}$. Figure 2a shows the printing area distribution and an estimation of the scanning time from the bottom to the top of the build. Since the machine constructor doesn't provide a value for the scanning time $(\mathrm{t})$, its estimation is obtained by multiplying the surface to be laser-scanned $(\mathrm{S})$ in each layer by the average scanning time per unit of surface (ST) calculated as:

$$
S T=\frac{1}{S S \times H D}
$$

with SS and HD indicating respectively the scanning speed and the hatch distance. However, the scanning time is also affected by both the geometry of the parts being printed, which determines the number of jump vectors (e.g. transition vectors where the laser is off), and the hard coded machine scanning delays. In order to take into account these influences a correction factor (f) is introduced in the calculation as shown in equation (4).

$$
t=f \times S \times S T
$$

Since the 5 levels of ED are obtained only by changing the laser power (see Table 1), ST assumes always the same value. Due to the simple geometries of the specimens and sacrificial parts, a heuristic value equal to 1.25 was assumed in the calculation. The choice of this particular value is based on a numerical simulation of the production process of these specimens. 
During the printing, the tensile bars were laser-scanned before the sacrificial parts, meaning that the amount of coalescence in the tensile bars is variable along the height depending on the relative area of the sacrificial parts (and inter-layer time). For the remainder of the article, the zones of constant extra printing area are referred to using the added area of the sacrificial parts (i.e. the 0 , $50,100,150$ and 200 zones).

\section{Table 1}

Figure 2

\subsection{Computed Tomography}

Computed Tomography (CT) was used to study the local porosity content in each of the five zones of the specimens produced at the five ED levels. All CT scans were performed using a $225 \mathrm{kV} \mathrm{CT}$ machine from Nikon Metrology using a Molybdenum target, a voltage of $110 \mathrm{kV}$, a current of 127 $\mu \mathrm{A}$ and 3000 projections. The magnification used was $x 10$, yielding a voxel size of $20 \mu \mathrm{m}$ which, according to Dewulf, Pavan et al. [6, 265], ensures a sufficiently accurate porosity measurement. The datasets were reconstructed using CT Pro 3D (Nikon Metrology) and analyzed using VGstudio max v.2.2 (Volume Graphics), where the closed porosity is calculated using the defect detection module, measuring pores which contained a minimum of 8 voxels. Consequently, the minimum measurable pore volume is $6.4 \cdot 10^{-05} \mathrm{~mm}^{3}$, while the minimum measurable pore sectional area (4 pixels) on a slice is $1.6 \cdot 10^{-03} \mathrm{~mm}^{2}$. By performing image processing of the CT-slices following the method described in [6], the porosity distribution along the printing direction was obtained.

\subsection{Surface Roughness}

The surface roughness of each tensile bar was measured using a Taylor Hobson Form Talysurf 120L surface analyzer for which the manufacturer ensures a measurement accuracy of $0.8 \mu \mathrm{m}$. This device works with a $120 \mathrm{~mm}$ traverse unit with $\mathrm{HeNe}$ laser interferometric transducer (Phase Grating Interferometer). The surface profile of each sample was acquired along three parallel 
tracks of $70 \mathrm{~mm}$ length with a spatial resolution of $0.25 \mu \mathrm{m}$ using a tip radius of $2 \mu \mathrm{m}$. The entire gauge length is contained in the middle of each track.

\subsection{Dimensional accuracy}

The local dimensional accuracy of all produced LS-PA12 parts was measured using fringe projection (GOM ATOS Compact Scan 2M, measuring volume 30 x 35 mm, $10 \mu \mathrm{m}$ resolution) in order to determine the influence of the inter-layer time on the local geometrical deviations. The width $w$, thickness $t$ and cross-sectional area $A$ of each of the 5 zones were averaged over the length of each respective zone. The dimensional deviations with respect to the CAD model were checked by fitting the scanned geometry to the CAD model by Gaussian minimization. Combining the information from the porosity measurements and fringe projection, the porosity corrected cross-sectional area $A^{\prime}$ can be computed as:

$$
A^{\prime}=A \times(1-P)
$$

with $A$ the measured cross-sectional and $P$ the porosity in the part.

\subsection{Mechanical testing}

Mechanical tests were performed displacement-control in a temperature-controlled lab environment on an Instron $4467(30 \mathrm{kN}$ load cell, $2 \mathrm{~mm} / \mathrm{min}$ strain rate crosshead displacement rate). Additionally, Digital Image Correlation (DIC) was performed using MatchID [276]. The parameters that wereused for the correlation algorithm are listed in Table 2. A complete survey of these parameters can be found in [276].

\section{Table 2}

\subsection{Thermal analysis}

After mechanical testing, selected samples from the different zones of the tensile bars produced at 25,35 and $45 \mathrm{~mJ} / \mathrm{mm}^{2}$ were subjected to thermal analysis by differential scanning calorimetry (DSC). The samples were heated from room temperature to $280{ }^{\circ} \mathrm{C}$ at a rate of $10{ }^{\circ} \mathrm{C} / \mathrm{min}$ on a DSC Q200 (TA Instruments). From the heat flow signal, the thermal transitions (glass transition and melt trajectory) can be studied. With the Universal Analysis software (TA Instruments), the 
enthalpy of fusion was calculated with a linear baseline between $140{ }^{\circ} \mathrm{C}$ and $200{ }^{\circ} \mathrm{C}$. The percentage crystallinity $(\% \mathrm{X})$ of the samples was then determined as the ratio between the enthalpy of fusion $\left(\Delta H_{m}\right)$ and the theoretical enthalpy of fusion for $100 \%$ crystalline LS-polyamide-12 $(209.3 \mathrm{~J} / \mathrm{g})$ [20 19] as in equation (6 4) below.

$$
\% X=\frac{\Delta H_{m}}{209.3 \mathrm{~J} / g}
$$

The percentage crystallinity for the different zones was averaged over three respective measurements on samples from three tensile bars and repeated for different ED levels.

\section{Results and Discussion}

\subsection{Porosity}

Figure 3 shows the average porosity of each layer along the gauge length of a tensile bar produced with an ED equal to $35 \mathrm{~mJ} / \mathrm{mm}^{2}$. The porosity variation among layers belonging to the same region is within the normal porosity fluctuation already reported in previous research $[6,9]$. This can be attributed to local variations of the powder packing density or to the different direction of the laservectors resulting from the alternate $x-y$ cross-scanning pattern. Moreover, a sudden change in the porosity level is clearly visible during the transition between zones of the gauge length which corresponds to different inter-layer times. Figure 4 shows the average porosity for each of the five regions of the five groups of tensile bars produced with different ED levels. For all the ED levels, the graph shows a decrease of the porosity content when the extra surface increases, which can be attributed to the extra time given to the polymer to flow and coalesce, which according to the Frenkel's model (2) should lead to a lower porosity level. However, the porosity-decreasing trend shows a deviation in the zone that corresponds to an additional surface of $50 \mathrm{~cm}^{2}$, it is not fully clear what is the mechanism behind this deviation.

\section{Figure 3}

\section{Figure 4}

\subsection{Surface roughness}


As described in section 2.3, the roughness measurement of each sample was performed on three parallel tracks, each with a length of $70 \mathrm{~mm}$. For a typical LS PA12 sample, the average Ra value is above $10 \mu \mathrm{m}$. Therefore, a cut-off wavelength $(\lambda \mathrm{c})$ of $8 \mathrm{~mm}$ is required per the ISO 4288-1996 standard to differentiate between waviness and roughness of the sample. For an accurate statistical calculation of the roughness, the minimum length of the track has to be equal to at least five times the cut-off wavelength, namely $40 \mathrm{~mm}$ for the samples examined.

\section{Figure 5}

Figure 5a shows two surface primary profiles of tensile bars printed with an ED equal to, respectively, $25 \mathrm{~mJ} / \mathrm{mm}^{2}$ and $45 \mathrm{~mJ} / \mathrm{mm}^{2}$. These profiles were acquired along paths parallel to the main axis of the tensile bars, with the gauge length located in the middle (approximately from 10 to $60 \mathrm{~mm}$ ). The two profiles refer to the lowest and highest ED levels used in the experiment. The surface profile related to the highest ED $\left(45 \mathrm{~mJ} / \mathrm{mm}^{2}\right)$ shows a larger variation in the transition between different zones of the gauge length. The wavelength corresponding to such variation is, anyway, above the cut-off wavelength $\lambda \mathrm{c}$, so it is not taken into account in the calculation of the roughness, but only in the waviness. The variation of the surface profiles corresponding to short wavelengths (below the cut-off wavelength $\lambda c$ ), namely those used in the calculation of the roughness, look similar in the two profiles. Figure $5 \mathrm{~b}$ shows other two surface primary profiles of specimens produced with an ED equal to, respectively, $30 \mathrm{~mJ} / \mathrm{mm}^{2}$ and $40 \mathrm{~mJ} / \mathrm{mm}^{2}$. Similar conclusion can be made also for these samples. Table 3 reports the mean Ra and Rz (DIN) values for the different ED levels, showing insignificant variation in the surface roughness among the different ED levels. The minimum required length of the track being larger than the size of each zone of the gauge length, only values for the different ED values are provided.

\section{Table 3}

he results reported in Table 3 and Figure 5 show how the combination of different EDs and inter layer times can affect locally the dimensions of the part locally, without affecting much the surface roughness. This concept will be further discussed in section 3.3 .

\subsection{Dimensions}


Figure 6 shows the dimensional deviations with respect to the CAD geometry of one sample per ED level. As a first conclusion, the five zones with increasing scanning time are clearly distinguishable for the specimens produced with an ED of $45 \mathrm{~mJ} / \mathrm{mm}^{2}$. In these samples, the deviation with respect to the CAD geometry is minimal $(0 \mathrm{~mm})$ in the 0-zone and it increases proportionally to the additionally scanned surface in the other zones. Secondly, the influence of the additional area that was scanned is proportional to the ED used to produce the samples. In fact, the $25 \mathrm{~mJ} / \mathrm{mm}^{2}$ specimens show no significant deviation between the regions of the tensile bar corresponding to different inter-layer times, while it is maximal for the one produced with an ED of $45 \mathrm{~mJ} / \mathrm{mm}^{2}$.

\section{Figure 6}

Figure 7 shows the cross-sectional area, averaged over the entire zone with a constant inter-layer time, as a function of the additional scanned area, for parts produced using different ED values. As a first conclusion, it is noted that a higher ED value leads to a higher average cross-sectional area over the gauge length of the specimen. This is due to the additional energy that is put into the material at a higher ED. As is also visible in Figure 6, the influence of the additional scanned area on the cross-sectional area of the tensile specimen is highest for the $45 \mathrm{~mJ} / \mathrm{mm}^{2}$ samples, and decreases proportional to the ED level. The $40 \mathrm{~mJ} / \mathrm{mm}^{2}$ and $45 \mathrm{~mJ} / \mathrm{mm}^{2}$ samples show the steepest decrease in cross-sectional area when moving from the 0-zone to the 50-zone, while no significant decrease is noticeable on average above $100 \mathrm{~cm}^{2}$ of extra surface. A possible explanation for this phenomenon is that, for the 0-zone, inter-layer times are comparably short, whereas the energy input is the highest in all samples produced. This gives rise to a local increase in temperature, as the material has less time to cool down, leading to an extra sintering of particles close to the surface. When the inter-layer time increases, the heat accumulation diminishes as the material has more time to cool down. Considering the $25 \mathrm{~mJ} / \mathrm{mm}^{2}$ and $30 \mathrm{~mJ} / \mathrm{mm}^{2}$ samples, only a slight decrease in the resulting cross-sectional area is noted due to the increase in additional scanned area, as the heat accumulation is not sufficient to induce extra sintering of the powder particles. The cross-sectional area of the LS-PA12 parts is thus strongly dependent on both the ED level used in the process and the inter-layer time. This is attributed to the combined effect of the two 
determining the local temperature during the process, and hence the amount of additionally sintered PA-12 particles. Moreover the results reported in Table 3 and Figure 5 show how the combination of different EDs and inter-layer times can affect locally the dimensions of the part, without affecting the surface roughness significantly.

\section{Figure 7}

\subsection{Thermal analysis}

The melting peak temperature does not vary significantly over the samples, and averages around $184.2 \pm 1.4{ }^{\circ} \mathrm{C}$. Figure 8 shows the degree of crystallinity in the different zones of samples produced at 25,35 and $45 \mathrm{~mJ} / \mathrm{mm}^{2}$, calculated using the enthalpy of melting. It can be seen that the degree of crystallinity does not vary much overall, with an average around $33.7 \%$ for all the samples. The degree of crystallinity increases slightly with decreasing ED, which is due to the higher crystallinity of the unmolten powder cores. This corresponds to the findings of Zarringhalam et al. [19 18]. The higher heat input by the laser results in less variation caused by the inter-layer time. At $45 \mathrm{~mJ} / \mathrm{mm}^{2}$, the heat input of the laser penetrates to the layer below sufficiently to remelt it. In that way, it removes the effect of the inter-layer time. The inter-layer time slightly affects the degree of crystallinity for the samples produced with 25 and $35 \mathrm{~mJ} / \mathrm{mm}^{2}$, resulting in a maximum degree of crystallinity for the 100-zone samples. This maximum in crystallinity is due to the interplay of two mechanisms: the coalescence and the cooldown of the exposed area. These two mechanisms are both influenced by the inter-layer time. With increasing inter-layer time, the time for the molten particles to coalesce with the adjacent particles increases. This provides more time for the heat to be distributed over the exposed area and for the crystals to homogeneously grow. The counteracting mechanism is the cooldown: with increasing inter-layer time, the consolidated area cools down by the atmosphere. This cooldown can result in the formation of an amorphous zone in the newly formed layer, which is not remolten by heat input of the next layer. This cooldown mechanism causes the crystallinity decrease observed at higher inter-layer times. For the inter-layer time resulting from $100 \mathrm{~cm}^{2}$ of additional scanned area, the interplay of these two effects (coalescence and cooldown) results in the maximum crystallinity. The deviations in the crystallinity values can be attested to both differences in sampling and 
differences of the material in the different zones. Cooling times are also dependent on the position of the parts within the powder bed. This results in an inhomogeneous annealing effect for (zones of the) parts within the build [7]. In that respect, the observed variations in crystallinity caused by varying inter-layer time is, therefore, minor.

Figure 8

3.5.Mechanical response

Table 4 shows the ultimate tensile strength $\left(\sigma_{U T S} \sigma_{\digamma}\right)$ of the parts produced with different ED levels, as well as the porosity $(\rho)$, cross-sectional area $\left(A_{r}\right)$ and porosity-corrected cross-sectional area $\left(A_{r}^{\prime}\right)$ that were measured in the zone with a specific inter-layer time where the specimen broke. Specifically, $\sigma_{U T S}$ is computed using the porosity-corrected cross-sectional area that was measured in the area of the failure. This table shows that parts that were produced using a higher ED level show higher tensile strength.

Also from this table, it can be deduced that, for the $30 \mathrm{~mJ} / \mathrm{mm}^{2}$ to $45 \mathrm{~mJ} / \mathrm{mm}^{2}$ samples, a linear relationship between $\sigma_{r}$ and $A_{r}^{\prime}$ exists. This is due to the combined effect of the reduction of crosssection throughout the gauge length and the stress-concentrations that are induced by the pores. It is noteworthy that the parts that were produced at $25 \mathrm{~mJ} / \mathrm{mm}^{2}$ do not follow this linear relationship and show a considerably lower tensile strength compared to the specimens produced at the other ED levels. This effect cannot solely be explained by the locally lower porosity corrected crosssectional area, but is attributed to incomplete sintering of the powder particles at this ED level [198], which led to more layered porosity, as previously reported by the authors in [7].

\section{Table 4}

Table 5 shows all the values of $A^{\prime}$ together with the zone of failure. Concerning the $25 \mathrm{~mJ} / \mathrm{mm}^{2}$, the failure mostly occurred in the zone having minimal $A^{\prime}$. Discrepancies hereto are linked to the high anisotropy of the samples due to the layered porosity as described above. Table 5 shows that, for the $40 \mathrm{~mJ} / \mathrm{mm}^{2}$ and $45 \mathrm{~mJ} / \mathrm{mm}^{2}$ parts, the lowest $A^{\prime}$ is present either in the 150 -zone or in the 
200-zone. However, these specimens consistently broke in the 200-zone. A contribution to this predominant localized failure comes from the sudden change in cross section between the region right before the gauge length and the 200-zone. Figures 5 and 6 clearly show this sudden shape deviation, which results in a stress concentration in the 200-zone. The same mechanism, with reduced magnitude, is also applicable to samples produced with an ED of $30 \mathrm{~mJ} / \mathrm{mm}^{2}$ and 35 $\mathrm{mJ} / \mathrm{mm}^{2}$. In fact, for these samples, the failure occurred in the 100- and 150-zones, which are the ones having the lowest $A^{\prime}$. From the previous argument, it can be understood that the increase in inter-layer time gives rise to local changes $A^{\prime}$ by both influencing the cross-sectional area of the part itself in se, as well as the local porosity content in the part. This, in turn, influences the location of failure in the parts, as mostly all parts failed in the zones where a combination of low $A^{\prime}$ and higher stress concentration were present.

\section{Table 5}

Finally, Figure 9 shows the strain fields that were measured by DIC. The illustrated measurements correspond to stress levels right before the failure of each specimen (46 MPa, $43 \mathrm{MPa}$ and $34 \mathrm{MPa}$ for, respectively, the $45 \mathrm{~mJ} / \mathrm{mm}^{2}, 35 \mathrm{~mJ} / \mathrm{mm}^{2}$ and $25 \mathrm{~mJ} / \mathrm{mm}^{2}$ specimens), as differences in elastic strains between the different zones of constant inter-layer time are located below the noise threshold of the employed DIC set-up, indicating a low effect of $A^{\prime}$ in the elastic regime. From this figure, a clear variation in the strain response within the components exists for the components that are produced using the different ED levels. This is especially true for the $45 \mathrm{~mJ} / \mathrm{mm}^{2}$, which is explained by the fact that the differences in $A^{\prime}$ are the most pronounced for these parts (see Video_ED45_Eyy). For the $25 \mathrm{~mJ} / \mathrm{mm}^{2}$ samples on the other hand, the variation of strain within the components is less pronounced, which also correlates with $A^{\prime}$. The presented strain measurements serve, therefore, as a further qualitative illustration of the effect of the inter-layer time on the mechanical response of PA12 parts produced via Laser Sintering. As such, when producing functional components for end-use in demanding applications, the effect of the interlayer time on the mechanical performance should be accounted for in either the process planning (e.g., by correct placement of different specimens in the build volume) or the design of the component (e.g. local changes in wall thickness or model topology), as indicated by the higher 
probability of rupture in zones with a higher inter-layer time, and reduced stiffness in the plastic regime. A critical side note hereto is that most end-use specimens are not used in the plastic regime in regular operational service, whereas no direct effect in the elastic regime was measured in the present study. Next to the mechanical performance, also the effect on the dimensional accuracy is non-trivial, as indicated in section 3.3, which serves as an additional argument for taking the EDlevel and inter-layer time into account.

Figure 9

\section{Conclusions}

This paper investigates the influence of the inter-layer time and energy density (ED) on criticalto-quality parameters like porosity content, crystallinity, dimensional accuracy and mechanical properties of selective laser sintered parts in polyamide. The gauge length of a standard tensile bar was divided into five regions for which different inter-layer times were used during production. This was done by adding a series of sacrificial parts around the tensile sample.

The porosity content is shown to be significantly affected by the inter-layer time, as indicated by the sudden changes in porosity at the transition between the different regions. Although the magnitude of the porosity content was also affected by the EDs used during the printing process, the dependency on the inter-layer times showed a similar behavior for all the ED levels tested. Also, the effect of the inter-layer time and ED on the dimensions of the tensile bars in the different regions of the gauge length is illustrated, with shorter times leading to larger dimensions and an increasing magnitude of the phenomena for higher ED's. The effect is believed to be linked to the different local temperatures during the process, with combinations of shorter inter-layer times and higher ED's leading to higher temperature and an increased heat flow in regions close to the surface, causing the sintering of more material. On the other hand, surface roughness was not significantly affected by neither the inter-layer time nor the ED.

Due to the variations of cross sectional area and porosity content of the different regions of the gauge length, the mechanical response was found to be not uniform along the gauge length of the tensile bar, with an increased intra-variability for higher ED values, leading to more localized strain during the tensile test. The location of the fracture regions of the tensile bars show good 
correlation with the porosity corrected cross sectional area, which is a metric including both the geometric deviation and the local porosity content.

The results gathered during this investigation show the importance of the combination of the interlayer time and ED used during the printing process in determining variation of important quality parameters like porosity, dimensions and mechanical response. Being able to ensure a more uniform inter-layer time during the process would allow significant reduction in the variation in quality of products produced via Laser Sintering. The findings are also relevant in an industrial context, where nowadays the standard production of both functional and esthetic parts is driven by an optimal filling of the build chamber of the machine. This affects, as indicated by this study, some important CTQ and should, therefore, not be ignored.

\section{Acknowledgements}

The authors would like to thank Bart Boeckmans from the metrology group of the mechanical engineering department of KU Leuven for his help with the surface roughness measurements. This work was supported by the European Union's Seventh Framework Program in the frame of the MSCA INTERAQCT project [grant number 607817]; the VLAIO in the TETRA project AMPLAMO [grant number HBC.2016.0081]; and the Flanders Innovation and Entrepreneurship (VLAIO), Strategic Initiative for Materials in Flanders (SIM), program STREAM, project POLYFORCE.

\section{Bibliography}

[1] D.L. Bourell, T.J. Watt, D.K. Leigh, B. Fulcher. (2014). Performance limitations in polymer laser sintering. Physics Procedia. Vol.56 pp.147-156. doi:10.1016/j.phpro.2014.08.157

[2] J.P. Kruth, P. Mercelis, J. Van Vaerenbergh, L. Froyen, M. Rombouts. (2005). Binding mechanisms in selective laser sintering and selective laser melting. Rapid Prototyping Journal. Vol. 11 Iss:1 pp. 26-36. doi:10.1108/13552540510573365.

[3] J.P. Kruth, G. Levy, F. Klocke, T.H.C. Childs. (2007). Consolidation phenomena in laser and powder-bed based layered manufacturing. CIRP Annals - Manufacturing Technology. Vol. 56 Iss:2 pp.730-759. doi:10.1016/j.cirp.2007.10.004.

[4] J.C. Nelson. (1993). Selective Laser Sintering: A Definition of the Process and an Empirical Sintering Model. University of Texas at Austin.

[5] R.D. Goodridge, C.J. Tuck, R.J.M. Hague. (2012). Laser sintering of polyamides and other polymers. Progress in Material Science. Vol.57 Iss:2 pp.229-267. doi:10.1016/j.pmatsci.2011.04.001. 
[6] W. Dewulf, M. Pavan, T. Craeghs, J.P. Kruth. (2016). Using X-ray Computed Tomography to improve the porosity level of polyamide-12 laser sintered parts, CIRP Annals - Manufacturing Technology. Vol.65 Iss:1 pp.205-208. doi:10.1016/j.cirp.2016.04.056.

[7] M. Pavan, T. Craeghs, P. Van Puyvelde, J.P. Kruth, W. Dewulf. (2016). Understanding the link between process parameters, microstructure and mechanical properties of laser sintered PA12 parts through X-ray computed tomography. $2^{\text {nd }}$ Pro-AM conference. Singapore, 16-19 May.

[8] J. Frenkel. (1945). Viscous flow of crystalline bodies under the action of surface tension. J Phys. Vol.9 Iss:5 pp.358-91.

[9] M. Pavan, T. Craeghs, R. Verhelst, O. Ducatteeuw, J.P. Kruth, W. Dewulf. (2016). CTbased quality control of Laser Sintering of Polymers. Case Studies in Nondestructive Testing and Evaluation. Vol.6 Part.B pp.62-68. doi:10.1016/j.csndt.2016.04.004.

[10] B. Caulfield, P.E. McHugh, S. Lohfeld. (2007). Dependence of mechanical properties of polyamide components on build parameters in the SLS process. Journal of Materials Processing Technology. Vol.182 Iss:1-3 pp.477-488. doi:10.1016/j.jmatprotec.2006.09.007.

[11] B. Van Hooreweder, J.P. Kruth. (2014). High cycle fatigue properties of selective laser sintered parts in polyamide 12. CIRP Annals-Manufacturing Technology. Vol.63 Iss:1 pp.241244. doi:10.1016/j.cirp.2014.03.060

[12] B. Van Hooreweder, D. Moens, R. Boonen, J.P. Kruth, P. Sas. (2013). On the difference in material structure and fatigue properties of nylon specimens produced by injection molding and selective laser sintering. Polymer Testing. Vol.32 pp.972-981. doi:10.1016/j.polymertesting.2013.04.014

[13] Van Hooreweder B, De Coninck F, Moens D, Boonen R, Sas P (2010) Microstructural characterization of SLS-PA12 Specimens Under Dynamic Tension/Compression Excitation. Polymer Testing 29(3):319-326.

[143] S. Griessbach, R. Lach, W. Grellmann. (2010). Structure-property correlations of laser sintered nylon 12 for dynamic dye testing of plastic parts. Polymer Testing. Vol.29 Iss:8 pp.10261030. doi:10.1016/j.polymertesting.2010.09.010.

[154] T.L. Starr, T.J. Gornet, J.S. Usher. (2011). The effect of process conditions on mechanical properties of laser-sintered nylon. Rapid Prototyping Journal. Vol.17 Iss:6 pp.418-423. doi: $10.1108 / 13552541111184143$.

[165] J.S. Usher, T.J. Gornet, T.L. Starr. (2013). Weibull growth modelling of laser sintered nylon 12. Rapid Prototyping Journal. Vol.19 Iss:4 pp.300-306. doi: 10.1108/13552541311323308

[176] M. Faes, Y. Wang, P. Lava, D. Moens. (2015). Variability in the mechanical properties of Laser Sintered PA-12 components. Proceedings 26th Solid Freeform Fabricattion Symposium. pp. 847-856. Austin, Texas, USA.

[187] M. Faes, Y. Wang, P. Lava, D. Moens. (2016). Variability, heterogeneity, and anisotropy in the quasi-static response of laser sintered PA12 components. Strain. doi: 10.1111/str.12219. 
[198] H. Zarringhalam, N. Hopkinson, N.F. Kamperman, J.J. Vlieger. (2006). Effects of processing on microstructure and properties of SLS Nylon 12. Materials Science and Engineering: A. Vol.435-436 pp.172-180. doi: 10.1016/j.msea.2006.07.084

[2019] S. Gogolewski, K. Czerniawska, M. Gasiorek. (1980). Effect of annealing on thermal properties and crystalline structure of polyamides. Nylon 12 (polylaurolactam). Colloid \& Polymer Science. Vol.258 Iss:10 pp.1130-1136. DOI: 10.1007/BF01382456.

[210] L. Verbelen, S. Dadbakhsh, M. Van den Eynde, J.P. Kruth, B. Goderis, P. Van Puyvelde. (2016). Characterization of polyamide powders for determination of laser sintering processability. European Polymer Journal. Vol.75 pp.163-174. doi:10.1016/j.eurpolymj.2015.12.014

[22t] R.J. Wang, L. Wang, L. Zhao, L. Zijian. (2007). Influence of process parameters on part shrinkage in SLS The International Journal of Advanced Manufacturing Technology. Vol. 33 Iss:5 pp.498-504. doi: 10.1007/s00170-006-0490-х

[23z] G. Strano, L. Hao, R.M. Everson, K.E. Evans. (2011). Multi-objective optimization of selective laser sintering processes for surface quality and energy saving. Proceedings of the Institution of Mechanical Engineers, Part B: Journal of Engineering Manufacture. Vol.225 Iss:9 pp.1673-1682. doi: 10.1177/0954405411402925

[243] C. C. Seepersad, T. Govett, K. Kim, M. Lundin, D. Pinero. (2012). A designer's guide for dimensioning and tolerancing sls parts. Proceedings 23rd Solid Freeform Fabricattion Symposium. pp. 921-931. Austin, Texas, USA.

[254] http://www.materialise.com/en/software/magics/modules/sinter-module accessed 14th March 2017

[265] M. Pavan, T. Craeghs, J.P. Kruth, W. Dewulf. (2017). Investigating the influence of X-ray CT parameters on porosity measurement of laser sintered PA12 using a design-of-experiment approach. [JOURNAL PUBBLICATION SUBMITTED]

[276] P. Lava, S. Cooreman, S. Coppieters, M. De Strycker, D. Debruyne. (2009). Assessment of measuring errors in DIC using deformation fields generated by plastic FEA. Optics and Lasers in Engineering. Vol.47 Iss:7-8 pp.747-753. doi:10.1016/j.optlaseng.2009.03.007. 
Figure 1: Schematic of a typical layout of a laser sintering system [2].

Figure 3: a) Printed surface distribution (left y-axis) and estimated scanning time (right y-axis) along the build height (x-axis); b) Build configuration with the tensile bars in the center and the sacrificial parts in the corners.

Figure 3: Porosity distribution for each printed layer from bottom to top of the gauge length of a tensile bar printed with an energy density equals to $35 \mathrm{~J} / \mathrm{mm}^{2}$.

Figure 4: Average porosity content of each region of the gauge length of the tensile bar for the five different ED levels. The confidence bounds indicate one standard deviation.

Figure 5: Surface primary profiles (no data filtering) along the length of tensile bars produced with ED equal to a) $25 \mathrm{~mJ} / \mathrm{mm}^{2}$ and $45 \mathrm{~mJ} / \mathrm{mm}^{2}$, and b) $30 \mathrm{~mJ} / \mathrm{mm}^{2}$ and $40 \mathrm{~mJ} / \mathrm{mm}^{2}$. The profiles are parallel to the main axis of the tensile bar and include the gauge length (approximately from 10 to $60 \mathrm{~mm}$ in the graph).

Figure 6: Deviation of the geometry of the produced samples with respect to the CAD geometry. The areas in the parts where no measurement data are present, are caused by markings put on the specimens to discern the different zones uniquely.

Figure7: Cross-sectional area as a function of the area of the sacrificial parts for the specimens produced at the different ED levels. The reported values are averaged over the respective zone. The confidence bounds indicate one standard deviation.

Figure 8 Degree of crystallinity for the 5 zones of the tensile bars produced at 25, 35 and 45 $\mathrm{mJ} / \mathrm{mm}^{2}$ energy densities. The confidence bounds indicate one standard deviation.

Figure 9: Longitudinal strain fields in a $45 \mathrm{~mJ} / \mathrm{mm}^{2}, 35 \mathrm{~mJ} / \mathrm{mm}^{2}$ and $25 \mathrm{~mJ} / \mathrm{mm}^{2}$ samples. 
Table 1: Laser scanning parameters that were used to produce the tensile bars at the five energy density levels

\begin{tabular}{ccccc}
\hline $\begin{array}{c}\text { ID Parameter } \\
\text { Set }\end{array}$ & Laser Power $(\mathbf{W})$ & Scan Speed $(\mathbf{m m} / \mathbf{s})$ & $\begin{array}{c}\text { Sean Spacing } \\
\text { Hatch distance } \\
(\mathbf{m m})\end{array}$ & $\begin{array}{c}\text { Energy Density } \\
\left(\mathbf{m} \mathbf{J} / \mathbf{m m}^{2}\right)\end{array}$ \\
\hline ED25 & 22.5 & 3000 & 0.3 & 25 \\
ED30 & 27.0 & 3000 & 0.3 & 30 \\
ED35 & 31.5 & 3000 & 0.3 & 35 \\
ED40 & 36.0 & 3000 & 0.3 & 40 \\
ED45 & 40.5 & 3000 & 0.3 & 45 \\
\hline
\end{tabular}


Table 2: Parameters that were employed for the DIC algorithm

\begin{tabular}{cc}
\hline DIC Specification & Value \\
\hline Camera noise & $0.35 \%$ \\
Matching criterion & ZNSSD \\
Prefiltering & Gaussian \\
Interpolation & Cubic spline \\
Subset size & $41 \mathrm{px}$ \\
Step size & $3 \mathrm{px}$ \\
Strain window & $21 \mathrm{px}$ \\
Displacement resolution & $1.73 \cdot 10^{-02} \mathrm{~mm}$ \\
Strain Resolution & $400 \mu \mathrm{m} / \mathrm{m}$ \\
\hline
\end{tabular}


Table 3: Mean Ra, Rz (DIN) and relative standard deviations of each group of tensile bars produced with the same ED.

\begin{tabular}{ccccc}
\hline $\begin{array}{c}\text { ID Parameter } \\
\text { Set }\end{array}$ & $\begin{array}{c}\text { Mean Ra } \\
(\boldsymbol{\mu m})\end{array}$ & $\begin{array}{c}\text { Standard } \\
\text { Deviation Ra }(\boldsymbol{\mu m})\end{array}$ & $\begin{array}{c}\text { Mean Rz (DIN) } \\
(\boldsymbol{\mu m})\end{array}$ & $\begin{array}{c}\text { Standard } \\
\text { Deviation Rz } \\
(\mathbf{D I N})(\boldsymbol{\mu m})\end{array}$ \\
\hline ED25 & 16.34 & 0.43 & 113.46 & 5.28 \\
ED30 & 14.56 & 1.34 & 102.30 & 10.77 \\
ED35 & 15.07 & 0.83 & 106.58 & 7.09 \\
ED40 & 16.11 & 1.42 & 112.46 & 8.02 \\
ED45 & 17.21 & 2.04 & 113.81 & 15.81 \\
\hline
\end{tabular}


Table 4: Ultimate tensile strength $\left(\sigma_{U T S} \sigma_{\bar{F}}\right)$, cross sectional area $\left(A_{r}\right)$, porosity $(\rho)$ and corrected crosssectional area $\left(A_{r}^{\prime}\right)$ in the zone where the specimen failed. The suffix $r$ denotes that the reported values were taken in the zone of failure.

\begin{tabular}{|c|c|c|c|c|c|}
\hline & $25 \mathrm{~mJ} / \mathrm{mm}^{2}$ & $30 \mathrm{~mJ} / \mathrm{mm}^{2}$ & $35 \mathrm{~mJ} / \mathrm{mm}^{2}$ & $40 \mathrm{~mJ} / \mathrm{mm}^{2}$ & $45 \mathrm{~mJ} / \mathrm{mm}^{2}$ \\
\hline$\sigma_{U T S} \sigma_{\bar{F}}[M P a]$ & 36.2 & 45.0 & 47.1 & 49.2 & 50.6 \\
\hline St.dev. & 2.0 & 1.2 & 1.2 & 0.6 & 0.73 \\
\hline$\rho_{r}[\%]$ & 4.75 & 4.19 & 3.80 & 3.77 & 3.96 \\
\hline St. Dev. & 0.16 & 0.15 & 0.36 & 0.17 & 0.13 \\
\hline$A_{r}\left[\mathrm{~cm}^{2}\right]$ & 124.1 & 124.7 & 125.7 & 127.5 & 128.5 \\
\hline St. Dev. & 1.17 & 0.49 & 0.72 & 1.52 & 2.33 \\
\hline$A_{r}^{\prime}\left[\mathrm{cm}^{2}\right]$ & 118.2 & 119.5 & 121.0 & 122.7 & 123.4 \\
\hline St. Dev. & 1.2 & 0.4 & 0.5 & 1.4 & 2.313 \\
\hline
\end{tabular}


Table 5: Porosity corrected cross section for all the specimens and zones of the gauge length. The zone where the failure happened is indicated in red, as well as a number indicating the order of the zone in a scale from 1- minimal porosity corrected cross section to 5 - maximal porosity corrected cross section.

* The sample broke outside the gauge length

\begin{tabular}{|c|c|c|c|c|c|}
\hline ID sample & $\mathbf{0}$ & 50 & 100 & 150 & 200 \\
\hline 25_1* & 119,05 & 120,62 & 118,17 & 118,60 & 118,98 \\
\hline $25 \_2$ & $119,25-5$ & 119,20 & 117,46 & 118,99 & 118,80 \\
\hline $25 \_3$ & 119,74 & 118,14 & $117,98-1$ & 117,87 & 118,99 \\
\hline $25 \_4$ & $118,81-3$ & 119,23 & 118,62 & 118,06 & 119,05 \\
\hline $25 \_5$ & 118,14 & 117,28 & 116,65 & $116,64-1$ & 118,05 \\
\hline 30_1 & 121,83 & 121,43 & $120,08-1$ & 120,54 & 120,65 \\
\hline 30_2 & 121,08 & 120,00 & $119,64-2$ & 119,55 & 121,36 \\
\hline 30_3 & 119,37 & 119,83 & $119,09-1$ & 119,19 & 119,87 \\
\hline 30_4 & 121,45 & 120,41 & $119,30-2$ & 118,75 & 120,45 \\
\hline 30_5 & 121,23 & 120,21 & 119,15 & $119,15-1$ & 120,02 \\
\hline 35_1 & 121,84 & 121,84 & 120,53 & $120,61-2$ & 122,50 \\
\hline $35 \_2$ & 123,42 & 122,96 & $121,41-1$ & 121,71 & 121,90 \\
\hline $35 \_3$ & 123,00 & 121,98 & 120,30 & $120,55-2$ & 120,84 \\
\hline 35_4 & 124,06 & 122,21 & $121,37-2$ & 120,84 & 121,71 \\
\hline $35 \_5^{*}$ & 123,43 & 122,74 & 120,51 & 120,29 & 120,58 \\
\hline 40_1 & 124,61 & 124.29 & 122.69 & 122.01 & $122.31-2$ \\
\hline $40 \_2$ & 127,03 & 124,23 & 122,59 & $122,39-1$ & 122,54 \\
\hline $40 \_3$ & 125,04 & 123,11 & 121,27 & 121,58 & $121,03-1$ \\
\hline 40_4 & 125,19 & 123.99 & 122.46 & 122.04 & $123.08-3$ \\
\hline 40_5 & - & - & - & - & - \\
\hline $45 \_1$ & 127,99 & 124,83 & 123,16 & 121,63 & $122,03-2$ \\
\hline 45_2 & 127,63 & 126.20 & 122.94 & 122.86 & $122.15-1$ \\
\hline $45 \_3 *$ & 127,48 & 125,27 & 122,12 & 122,31 & 121,13 \\
\hline 45_4 & 128,48 & 126,76 & 123,15 & 123,07 & $122,02-1$ \\
\hline 45_5 & 128,45 & 125,36 & 123,50 & 121,85 & $122,79-2$ \\
\hline
\end{tabular}

